\title{
A NON-SMOOTH EXTENSION OF FRECHET DIFFERENTIABILITY OF THE NORM WITH APPLICATIONS TO NUMERICAL RANGES
}

\author{
by C. APARICIO, F. OCAÑA, R. PAYÁ and A. RODRÍGUEZ
}

(Received 17 January, 1985)

1. Introduction. The following result in the theory of numerical ranges in Banach algebras is well known (see [3, Theorem 12.2]). The numerical range of an element $F$ in the bidual $A^{\prime \prime}$ of a unital Banach algebra $A$ is the closure of the set of values at $F$ of the $w^{*}$-continuous states of $A^{\prime \prime}$. As a consequence of the results in this paper the following more general theorem is obtained.

(1) Let $A$ be a unital Banach algebra whose Banach space is the dual space of a Banach space. Then the numerical range of an element $a$ in $A$ is the closure of the set of values at a of the $w^{*}$-continuous states of $A$.

$W^{*}$-algebras and Bollobás extremal algebras [2,5] are examples of Banach algebras satisfying the assumption of the above theorem. In fact the associativity of the product is not needed, so the conclusion of the theorem is true for a wider class of algebras which includes for example $J B W$-algebras [13] and noncommutative $J W^{*}$-algebras [10].

The main results in this paper are obtained in the general context of numerical range spaces. Since the numerical range in a unital Banach algebra does not depend on the product but only on the underlying Banach space and on the unit, numerical ranges can be considered in an arbitrary Banach space $X$ once a norm-one element $u$ has been selected. The pair $(X, u)$ is called a numerical range space (see [9]). In this way our results are of a purely geometric nature.

We introduce the concept of strong numerical range space which is a natural non-smooth extension of Fréchet differentiability of the norm at a point in the unit sphere of a Banach space. We prove that our requirement on a numerical range space is implied by the norm-to-norm and implies the norm-to-weak upper semicontinuity of the duality mapping at the distinguished element. Both concepts of upper semicontinuity were introduced in [8]. The duality mapping of a unital complete normed nonassociative algebra $A$ is norm-to-norm upper semicontinuous at the unit [9], so $(A, I)(I$ the unit of $A)$ is a strong numerical range space. It was announced in [11] that the numerical range of a bounded function $\Phi$ from an arbitrary set $\Lambda$ into a unital complete normed nonassociative algebra $A$ is the closed convex hull of the union of the numerical ranges of.the elements $\Phi(\lambda)$. We prove (Theorem 2.7) that when an arbitrary numerical range space $(X, u)$ plays the role of $(A, I)$ the same statement is true for every set $\Lambda$ (equivalently for just one infinite set $\Lambda)$ if and only if $(X, u)$ is strong. The result announced in [11] follows as a very. particular case.

Glasgow Math. J. 28 (1986) 121-137. 
Our main result in $\S 3$ is the following.

(2) Let $X$ be a Banach space and $g$ a norm-one element in the unit sphere of the dual space $X^{\prime}$. Assume that $\left(X^{\prime}, g\right)$ is a strong numerical range space. Then the numerical range of any element $f$ in $X^{\prime}$ is the closure of the set of values at $f$ of the $w^{*}$-continuous states of $\left(X^{\prime}, g\right)$.

Note that (1) is a very particular case of (2). Also it is clear that under the assumptions of (2) the functional $g$ attains its norm, so by James's theorem (see [6]) we deduce that if $\left(X^{\prime}, g\right)$ is strong for all $g$ in the unit sphere of $X^{\prime}$ then $X$ is reflexive. This is a non-smooth extension of a well known result [6, Corollary 1, p. 34]. We prove in $\$ 4$ that the bidual space of a strong numerical range space is also strong (the distinguished element remains unchanged). It follows from this result and (2) that Fréchet differentiability of the norm of a Banach space at a point implies Fréchet differentiability of the bidual norm at the same point.

2. Strong numerical range spaces. By numerical range space we mean a pair $(X, u)$, where $X$ is a Banach space over the field $\mathbb{K}(\mathbb{R}$ or $\mathbb{C})$ and $u$ is a fixed element in the unit sphere $S(X)$ of $X$. We shall usually keep in mind the element $u$ and say that $X$ is a numerical range space. We define the state space $D(X)$ of $X$ to be the nonempty convex and $w^{*}$-compact subset of the dual space $X^{\prime}$ given by

$$
D(X)=\left\{f \in X^{\prime}:\|f\|=f(u)=1\right\} .
$$

The numerical range $V(X, x)$ (or $V(x)$ when $X$ is clear from the context) of an element $x$ in $X$ is defined by

$$
V(X, x)=\{f(x): f \in D(X)\} .
$$

We have clearly that $V(x)$ is a nonempty compact convex subset of $\mathbb{K}$ and

$$
\begin{aligned}
& |\lambda| \leqq\|x\| \text { for all } \lambda \text { in } V(x) \text { and } x \text { in } X . \\
& V(x+y) \subset V(x)+V(y) \text { for all } x, y \text { in } X . \\
& V(\lambda x+\eta u)=\lambda V(x)+\eta \text { for all } \lambda, \eta \text { in } \mathbb{K} \text { and } x \text { in } X .
\end{aligned}
$$

The real function $M$ defined on $X$ by

$$
M(x)=\operatorname{Max} \operatorname{Re} V(x) \quad(x \in X)
$$

is positively homogeneous, subadditive and bounded by 1 in the closed unit ball $B(X)$ of $X$ so it satisfies

$$
|M(x)-M(y)| \leqq\|x-y\| \text { for all } x, y \text { in } X .
$$

2.1. Definition. Let $(X, u)$ be a numerical range space and $S$ a nonempty bounded 
subset of $X$. We define a real function $\phi_{S}$ on $\mathbb{R}^{+}$by

$$
\phi_{S}(\alpha)=\operatorname{Sup}\left\{\frac{\|u+\alpha x\|-1}{\alpha}-M(x): x \in S\right\} \quad(\alpha>0) .
$$

We shall simply write $\phi$ for $\phi_{B(X)}$.

2.2. Proposition. Let $(X, u)$ be a numerical range space and $S$ a nonempty bounded subset of $X$.

(i) $\phi_{S}$ is a nonnegative, nondecreasing function, so $\phi_{S}$ has a limit at 0.

(ii) If $S$ is compact, then $\lim _{\alpha \rightarrow 0} \phi_{S}(\alpha)=0$.

Proof. (i) For every $x$ in $X$ the function $t \rightarrow\|u+t x\|(t \in \mathbb{R})$ is convex, so $\phi_{S}$ is the least upper bound of a family of nondecreasing functions. It is known that

$$
M(x)=\lim _{\alpha \rightarrow 0+} \frac{\|u+\alpha x\|-1}{\alpha}=\operatorname{Inf}\left\{\frac{\|u+\alpha x\|-1}{\alpha}: \alpha>0\right\}
$$

for all $x$ in $X$ (see [7, Theorem V.9.5]) and this shows that $\phi_{S}$ is nonnegative.

(ii) Apply Dini's theorem to the nonincreasing sequence $\left(f_{n}\right)$ of continuous functions on $X$ defined by

$$
f_{n}(x)=\|n u+x\|-n \quad(x \in X, n \in \mathbb{N}),
$$

which converges pointwise on the compact set $S$ to the continuous function $M$, to obtain that $\left(f_{n}\right)$ converges uniformly on $S$; that is $\left(\phi_{S}(1 / n)\right) \rightarrow 0$.

As a particular case of the above proposition, if $(X, u)$ is a finite-dimensional numerical range space we have $\lim _{\alpha \rightarrow 0} \phi(\alpha)=0$, where $\phi$ is the function defined in 2.1.

2.3. Definition. A numerical range space $(X, u)$ will be called strong when $\lim _{\alpha \rightarrow 0} \phi(\alpha)=0$. Equivalently, $(X, u)$ is strong when

$$
\lim _{\alpha \rightarrow 0+} \frac{\|u+\alpha x\|-1}{\alpha}=M(x) \text { uniformly in } B(X) .
$$

\subsection{EXAMPLES.}

(i) Let $X$ be a Hilbert space and $u \in S(X)$. For $x$ in $X$ and $\alpha>0$ we can write

$$
\|u+\alpha x\| \leqq \frac{1}{2}\left(\|u+\alpha x\|^{2}+1\right)=1+\alpha \operatorname{Re}(u \mid x)+\frac{\alpha^{2}}{2}\|x\|^{2},
$$

so we have

$$
\frac{\|u+\alpha x\|-1}{\alpha}-M(x)=\frac{\|u+\alpha x\|-1}{\alpha}-\operatorname{Re}(u \mid x) \leqq \frac{\alpha}{2}\|x\|^{2}
$$

and $\phi(\alpha) \leqq \alpha / 2$. This proves that $(X, u)$ is a strong numerical range space. 
(ii) Consider the numerical range space $\left(l^{1}(\mathbb{R}), u\right)$ where $u$ is the sequence $\left(1 / 2^{n}\right)$. Let $\left(x_{n}\right)$ be the sequence with -1 in the $n$th place and 0 elsewhere and $\alpha_{n}=1 / n$. We have

$$
\phi\left(\alpha_{n}\right) \geqq \frac{\left\|u+\alpha_{n} x_{n}\right\|-1}{\alpha_{n}}-M\left(x_{n}\right)=2-\frac{n}{2^{n-1}}
$$

so $\left(l^{1}(\mathbb{R}), u\right)$ is not strong.

2.5. REMARK. If $X$ is smooth at $u$ it is clear that $(X, u)$ is strong if and only if the norm of $X$ is Fréchet differentiable at $u$. So the concept of strong numerical range space is a non-smooth extension of Fréchet differentiability of the norm at a point in $S(X)$.

If $Y$ is a closed subspace of $X$ and $u$ belongs to $Y$ we have clearly $V(Y, y)=V(X, y)$ for all $y$ in $Y$; hence $(Y, u)$ is strong whenever $(X, u)$ is.

Given an arbitrary nonempty set $\Lambda$ and a numerical range space $(X, u)$ we denote by $(B(\Lambda, X), u)$ the numerical range space of bounded functions from $\Lambda$ into $X$, with the supremum norm, whose distinguished element (which we denote again by $u$ ) is the constant function equal to $u$ on $\Lambda$. If $(B(\Lambda, X), u)$ is strong for some nonempty set $\Lambda$, then so is $(X, u)$, since $X$ can be identified with the subspace of constant functions. On the other hand it is not difficult to verify that if $(X, u)$ is strong then so is $(B(\Lambda, X), u)$ for all $\Lambda$. When $\Lambda$ is a finite set one can verify that

$$
V(\Phi)=\operatorname{co} \bigcup_{\lambda \in \Lambda} V(\Phi(\lambda)) \text { for all } \Phi \text { in } B(\Lambda, X),
$$

where co denotes convex hull (see Corollary 2.10 below for a concrete proof). Also it is known that if $A$ is a power associative, unital, complete normed algebra and $\Lambda$ is any nonempty set then

$$
V(\Phi)=\overline{\operatorname{co}} \bigcup_{\lambda \in \Lambda} V(\Phi(\lambda)) \text { for all } \Phi \text { in } B(\Lambda, A)
$$

where $\overline{\text { co }}$ denotes closed convex hull [11, Proposition 3]. Our main result in this section (Theorem 2.7) states that the above property characterizes strong numerical range spaces.

2.6. Proposition. Let $(X, u)$ be a numerical range space and $\Lambda$ a nonempty set. Then for all $\Phi$ in $B(\Lambda, X)$ we have

$$
\overline{\operatorname{co}} \bigcup_{\lambda \in \Lambda} V(\Phi(\lambda)) \subset V(\Phi)
$$

Proof. For $\lambda$ in $\Lambda$ and $f$ in $D(X)$, the mapping $\Phi \rightarrow f(\Phi(\lambda))$ belongs to $D(B(\Lambda, X))$, so we have $V(\Phi(\lambda)) \subset V(\Phi)$ for all $\lambda$ in $\Lambda$. Now observe that $V(\Phi)$ is convex and compact.

2.7. TheOREM. Let $(X, u)$ be a numerical range space. The following statements are equivalent.

(a) $(X, u)$ is strong. 
(b) The formula

$$
V(\Phi)=\overline{\mathrm{co}} \bigcup_{\lambda \in \Lambda} V(\Phi(\lambda))
$$

holds for all nonempty sets $\Lambda$ and all $\Phi$ in $B(\Lambda, X)$.

(c) There is an infinite set $\Lambda$ such that

$$
V(\Phi)=\overline{\operatorname{co}} \bigcup_{\lambda \in \Lambda} V(\Phi(\lambda))
$$

for all $\Phi$ in $B(\Lambda, X)$.

(d) $V(\Phi)=\overline{\text { co }} \bigcup_{n \in \mathbb{N}} V(\Phi(n))$ for all $\Phi$ in $B(\mathbb{N}, X)$.

In the proof of the above result the following lemma will be needed.

2.8. LemMA. Let $(X, u)$ be a numerical range space, $S$ a balanced bounded nonempty subset of $X, \Lambda$ a nonempty set and $\Phi$ a function from $\Lambda$ into $X$ such that $\Phi(\Lambda) \subset S$. Assume that $\lim _{\alpha \rightarrow 0} \phi_{S}(\alpha)=0$. Then

$$
V(\Phi)=\overline{\operatorname{co}} \bigcup_{\lambda \in \Lambda} V(\Phi(\lambda)) .
$$

Proof. Fix $z$ in $S(\mathbb{K}), \alpha>0$ and $\lambda \in \Lambda$. Since $S$ is balanced we have $z \Phi(\lambda) \in S$ so

$$
\frac{1}{\alpha}(\|u+\alpha z \Phi(\lambda)\|-1) \leqq M(z \Phi(\lambda))+\phi_{s}(\alpha)
$$

and we deduce that

$$
\frac{1}{\alpha}(\|u+\alpha z \Phi\|-1) \leqq \operatorname{Sup}\{M(z \Phi(\lambda)): \lambda \in \Lambda\}+\phi_{S}(\alpha) \leqq M(z \Phi)+\phi_{S}(\alpha),
$$

where the last inequality holds by Proposition 2.6. Now let $\alpha \rightarrow 0$ to obtain

$$
M(z \Phi)=\operatorname{Sup}\{M(z \Phi(\lambda)): \lambda \in \Lambda\}
$$

that is

$$
\operatorname{Max} \operatorname{Re}[z V(\Phi)]=\operatorname{Max} \operatorname{Re}\left[z \overline{\operatorname{co}} \bigcup_{\lambda \in \Lambda} V(\Phi(\lambda))\right] .
$$

This holds for all $z$ in $S(\mathbb{K})$ and implies that the compact convex sets $V(\Phi)$ and $\overline{c o} \bigcup_{\lambda \in \Lambda} V(\Phi(\lambda))$ agree.

Proof of Theorem 2.7. (a) $\Rightarrow$ (b) We can clearly arrange $\|\Phi\| \leqq 1$ and apply the above lemma.

(b) $\Rightarrow$ (c) This is clear.

(c) $\Rightarrow$ (d) Let $\Lambda$ be the set appearing in (c) and $\sigma$ a mapping from $\Lambda$ onto $\mathbb{N}$. The 
mapping $\Phi \rightarrow \Phi \cdot \sigma$ from $B(\mathbb{N}, X)$ into $B(\Lambda, X)$ is linear, isometric and preserves the distinguished elements, so we have

$$
V(\Phi)=V(\Phi \cdot \sigma)=\overline{\mathrm{co}} \bigcup_{\lambda \in \Lambda} V(\Phi(\sigma(\lambda)))=\overline{\mathrm{co}} \bigcup_{n \in \mathbb{N}} V(\Phi(n)) .
$$

(d) $\Rightarrow$ (a) Suppose that $(X, u)$ is not strong. Then we can find $\varepsilon_{0}$ and sequences $\left(x_{n}\right)$ in $B(X)$ and $\left(\alpha_{n}\right)$ in $\mathbb{R}^{+}$satisfying

$$
\alpha_{n}<\frac{1}{n} \text { and } \frac{\left\|u+\alpha_{n} x_{n}\right\|-1}{\alpha_{n}}-M\left(x_{n}\right) \geqq \varepsilon_{0}
$$

for all $n$ in $\mathbb{N}$. Write $r=\overline{\lim } M\left(x_{n}\right)$ and find $p \in \mathbb{N}$ such that

$$
M\left(x_{n+p}\right)<r+\frac{\varepsilon_{0}}{2}
$$

holds for all $n$ in $\mathbb{N}$. Consider the mapping $\Phi: \mathbb{N} \rightarrow X$ given by

$$
\Phi(n)=x_{n+p} \quad(n \in \mathbb{N}) .
$$

We have

$$
\frac{\left\|u+\alpha_{n+p} \Phi\right\|-1}{\alpha_{n+p}} \geqq \frac{\left\|u+\alpha_{n+p} x_{n+p}\right\|-1}{\alpha_{n+p}} \geqq \varepsilon_{0}+M\left(x_{n+p}\right)
$$

and by taking upper limits $M(\Phi) \geqq r+\varepsilon_{0}$. Now we apply (d) to obtain

$$
r+\varepsilon_{0} \leqq M(\Phi)=\operatorname{Sup}\{M(\Phi(n)): n \in \mathbb{N}\}=\operatorname{Sup}\left\{M\left(x_{n+p}\right): n \in \mathbb{N}\right\} \leqq r+\frac{\varepsilon_{0}}{2},
$$

a contradiction.

We conclude this section with some other consequences of the arguments in the proof of the above theorem (mainly Lemma 2.8).

2.9. Proposition. Let $(X, u)$ be a numerical range space, $\Lambda$ a nonempty set and $\Phi$ a mapping from $\Lambda$ into $X$. Assume that $\Phi(\Lambda)$ is relatively compact in $X$. Then

$$
V(\Phi)=\overline{\operatorname{co}} \bigcup_{\lambda \in \Lambda} V(\Phi(\lambda))
$$

Proof. Let $S$ be the balanced hull of the closure of $\Phi(\Lambda)$. By Proposition 2.2 we have $\lim _{\alpha \rightarrow 0} \phi_{S}(\alpha)=0$. Now apply Lemma 2.8 . Then

2.10. Corollary. Let $(X, u)$ be a numerical range space and $\Lambda$ a finite nonempty set.

$$
V(\Phi)=\operatorname{co} \bigcup_{\lambda \in \Lambda} V(\Phi(\lambda))
$$

for every function $\Phi$ from $\Lambda$ into $X$. 
This corollary, together with Example 2.4(ii) and Theorem 2.7 show that the only sets $\Lambda$ such that

$$
V(\Phi)=\overline{\operatorname{co}} \bigcup_{\lambda \in \Lambda} V(\Phi(\lambda))
$$

holds for every numerical range space $(X, u)$ and all $\Phi$ in $B(\Lambda, X)$ are the finite sets.

The next corollary is well known but its deduction from Proposition 2.9 is particularly easy.

2.11. Corollary. Let $A^{b}(C, \mathbb{K})($ resp. $A(C, \mathbb{K}))$ the Banach space of bounded affine (resp. continuous affine) functions from a compact convex set $C$ into the scalar field $\mathbb{K}$ with the supremum norm. Then

(i) $V(\Phi)=\Phi(C)^{-}$for all $\Phi$ in $A^{b}(C, \mathbb{K})$.

(ii) $V(\Phi)=\Phi(C)$ for all $\Phi$ in $A(C, \mathbb{K})$.

Proof. Consider $A(C, \mathbb{K}) \subset A^{b}(C, \mathbb{K}) \subset B(C, \mathbb{K})$ as numerical range spaces (the distinguished element is always the function identically one). By Proposition 2.9 we have

$$
V(\Phi)=\overline{\mathrm{co}} \Phi(C) \text { for all } \Phi \text { in } B(C, \mathbb{K}) \text {. }
$$

For continuous functions on a compact set we do not need all their values in order to determine the numerical range of the function as we show below.

2.12. Corollary. Let $\Lambda$ be a Hausdorff compact topological space and $\Phi$ a continuous function from $\Lambda$ into an arbitrary numerical range space $(X, u)$. Let $\Gamma$ be a dense subset of $\Lambda$. Then

$$
V(\Phi)=\overline{\operatorname{co}} \bigcup_{\gamma \in \Gamma} V(\Phi(\gamma))
$$

Proof. By mapping each continuous function from $\Lambda$ to $X$ into its restriction to $\Gamma$ we obtain an isometric linear embedding of the continuous functions into $B(\Gamma, X)$. Now use Proposition 2.9 to obtain

$$
V(\Phi)=V\left(\Phi_{\mid \Gamma}\right)=\overline{c o} \bigcup_{\gamma \in \Gamma} V(\Phi(\gamma))
$$

3. Dual numerical range spaces. Let $X$ be a Banach space, $g$ a norm-one element in $X^{\prime}$ and consider the numerical range space $\left(X^{\prime}, g\right)$. We seek conditions on $\left(X^{\prime}, g\right)$ under which numerical ranges in $X^{\prime}$ can be determined by the $w^{*}$-continuous states of $X^{\prime}$ (that is by the elements in $\left.D\left(X^{\prime}\right) \cap X\right)$. No intrinsical condition on $X^{\prime}$ can be necessary and sufficient, as the following example shows. Consider the case $X^{\prime}=l^{1}$ and let $g$ be the sequence $\left\{1 / 2^{n}\right\}$. If we take $X=c_{0}$ there are no $w^{*}$-continuous states on $X^{\prime}$, while if we take $X=c$, then the unique state of $X^{\prime}$ is $w^{*}$-continuous. We prove in this section that if $\left(X^{\prime}, g\right)$ is strong, then numerical ranges in $X^{\prime}$ are determined by the $w^{*}$-continuous states, so giving a sufficient condition which does not involve the concrete predual under consideration.

Let $(X, u)$ be a numerical range space. An element $x$ in $X$ will be called dissipative 
whenever $\operatorname{Re} \lambda \leqq 0$ for all $\lambda$ in $V(x)$. We denote by $\operatorname{Dis}(X)$ the set of dissipative elements in $X$. $\operatorname{Dis}(X)$ is a closed convex cone.

3.1. Lemma. Let $(X, u)$ be a numerical range space and $f$ a linear functional on $X$. The following statements are equivalent.

(a) $\operatorname{Re} f(x) \leqq 0$ for all $x$ in $\operatorname{Dis}(X)$.

(b) There is a $\lambda \geqq 0$ such that $f \in \lambda D(X)$.

Proof. Assume that $f$ satisfies (a). For $x$ in $X$ we have $x-M(x) u \in \operatorname{Dis}(X)$ and $-u \in \operatorname{Dis}(X)$ so that

$$
\operatorname{Re} f(x) \leqq M(x) \operatorname{Re} f(u) \leqq\|x\| \operatorname{Re} f(u) .
$$

This shows that $\operatorname{Re} f$ is a real-linear continuous functional on $X$ with norm $\operatorname{Re} f(u)$, so $f \in X^{\prime}$ and $\|f\|=\operatorname{Re} f(u)$. From $|f(u)| \leqq \operatorname{Re} f(u)$ we deduce $f(u)=\operatorname{Re} f(u)=\|f\|$ and (b) follows with $\lambda=f(u)$. The implication $(b) \Rightarrow(a)$ follows directly from the definition of $\operatorname{Dis}(X)$.

3.2. Lemma. Let $(X, u)$ be a numerical range space, $\tau$ a locally convex Hausdorff topology on $X$ and denote by $X_{\tau}^{\prime}$ the space of $\tau$-continuous linear functionals on $X$. The following assertions are equivalent.

(a) $\operatorname{Dis}(X)$ is $\tau$-closed.

(b) $V(x)=\left\{f(x): f \in D(X) \cap X_{\tau}^{\prime}\right\}^{-}$for all $x$ in $X$.

(c) $D(X)=w^{*}-c l .\left(D(X) \cap X_{\tau}^{\prime}\right)$.

Proof. $(a) \Rightarrow(b)$ For $x$ in $X$ the set

$$
H_{x}=\left\{f(x): f \in D(X) \cap X_{r}^{\prime}\right\}^{-}
$$

is convex, compact and is included in $V(x)$. To prove (b) it is clearly enough to show that $M(x) \leqq \operatorname{Max} \operatorname{Re} H_{x}$.

If $\eta$ is a real number with $\eta<M(x)$ the element $x-\eta u$ is not dissipative, so by (a) and the Hahn-Banach theorem of separation of convex sets (see [1, Theorem 34.7 and Exercise 34.12]) there is $f$ in $X_{\tau}^{\prime}$ with $f \neq 0$ such that

$$
\operatorname{Re} f(y) \leqq \operatorname{Re} f(x-\eta u) \text { for all } y \text { in } \operatorname{Dis}(X) .
$$

Since $\operatorname{Dis}(X)$ is a cone we have

$$
\operatorname{Re} f(y) \leqq 0 \text { for all } y \text { in } \operatorname{Dis}(X)
$$

and by the above lemma we can suppose that $f$ is a state, so

$$
0 \leqq \operatorname{Re} f(x-\eta u)=\operatorname{Re} f(x)-\eta
$$

and we deduce $\eta \leqq \operatorname{Re} f(x) \leqq \operatorname{Max} \operatorname{Re} H_{x}$. Now let $\eta \rightarrow M(x)$ to obtain $M(x) \leqq$ $\operatorname{Max} \operatorname{Re} H_{x}$ as required.

(b) $\Rightarrow$ (c) Let $f \in D(X)$ be such that $f \notin w^{*}$-cl. $\left(D(X) \cap X_{\tau}^{\prime}\right)$. The above mentioned 
separation theorem gives an $x$ in $X$ such that

$$
\operatorname{Sup}\left\{\operatorname{Re} g(x): g \in w^{*}-c l .\left(D(X) \cap X_{\tau}^{\prime}\right)\right\}<\operatorname{Re} f(x) .
$$

This contradicts (b).

(c) $\Rightarrow$ (a) By (c) we have that an element $x$ in $X$ is dissipative if and only if $\operatorname{Re} f(x) \leqq 0$ for all $f$ in $D(X) \cap X_{\tau}^{\prime}$. This clearly implies that $\operatorname{Dis}(X)$ is $\tau$-closed.

3.3. Lemma. Let $(X, u)$ be a strong numerical range space. Then

$$
B(X) \cap \operatorname{Dis}(X)=B(X) \cap\left[\bigcap_{\alpha>0} B\left(-\frac{u}{\alpha}, \frac{1}{\alpha}+\phi(\alpha)\right)\right] .
$$

Proof. The inclusion

$$
B(X) \cap \operatorname{Dis}(X) \subset B(X) \cap\left[\bigcap_{\alpha>0} B\left(-\frac{u}{\alpha}, \frac{1}{\alpha}+\phi(\alpha)\right)\right]
$$

is true for every numerical range space. In fact if $x \in B(X)$ and $M(x) \leqq 0$ we have

$$
\frac{\|u+\alpha x\|-1}{\alpha} \leqq \frac{\|u+\alpha x\|-1}{\alpha}-M(x) \leqq \phi(\alpha) \quad(\alpha>0)
$$

that is,

$$
\left\|x+\frac{u}{\alpha}\right\| \leqq \frac{1}{\alpha}+\phi(\alpha) \text { for all } \alpha>0 .
$$

Now if $(X, u)$ is strong and $x \in B(X) \cap\left[\bigcap_{\alpha>0} B\left(-\frac{u}{\alpha}, \frac{1}{\alpha}+\phi(\alpha)\right)\right]$ we have

$$
M(x)=\lim _{\alpha \rightarrow 0+} \frac{\|u+\alpha x\|-1}{\alpha} \leqq \lim _{\alpha \rightarrow 0+} \phi(\alpha)=0,
$$

so that $x \in B(X) \cap \operatorname{Dis}(X)$. Now we put the pieces together and obtain the following result.

3.4. Theorem. Let $X$ be a Banach space, $g$ a norm-one element in $X^{\prime}$ and assume that the numerical range space $\left(X^{\prime}, g\right)$ is strong. Then

$$
V(f)=\{f(x): x \in S(X), g(x)=1\}^{-} \text {for all } f \text { in } X^{\prime} .
$$

Proof. In view of Lemma 3.3 we have that $B\left(X^{\prime}\right) \cap \operatorname{Dis}\left(X^{\prime}\right)$ is $w^{*}$-closed; hence so is $\operatorname{Dis}\left(X^{\prime}\right)$ by the Krein-Smulian theorem. Now apply Lemma 3.2 to the numerical range space $\left(X^{\prime}, g\right)$ with $\tau=w^{*}$ and take into account the identification of $\left(X^{\prime}\right)_{w^{*}}^{\prime}$ with $X$.

The following corollary is a non-smooth extension of the well known fact (see [6, Corollary 1, p. 34]) that every Banach space with Fréchet differentiable dual is reflexive.

3.5. Corollary. Let $X$ be a Banach space. Assume that for all $g$ in $S\left(X^{\prime}\right)$ the numerical range space $\left(X^{\prime}, g\right)$ is strong. Then $X$ is reflexive. 
Proof. By Theorem 3.4 every $g$ in $S\left(X^{\prime}\right)$ attains its norm. Now apply James's theorem (see [6, Theorem 3, p. 12]).

4. The bidual of a numerical range space. Let $(X, u)$ be a numerical range space. The canonical injection of $X$ into its bidual space $X^{\prime \prime}$ allows us to consider the numerical range space $\left(X^{\prime \prime}, u\right)$ which we call the bidual of $(X, u)$. The purpose of this section is to prove that if $(X, u)$ is strong then so is $\left(X^{\prime \prime}, u\right)$.

We need some previous lemmas in which the following notation will be used. For a subset $S$ of a Banach space $X$ we write

$$
S^{0}=\left\{f \in X^{\prime}: \operatorname{Re} f(x) \leqq 1 \text { for all } x \text { in } S\right\} .
$$

Also if $T$ is a subset of $X^{\prime}$ we write

$$
{ }^{0} T=\{x \in X: \operatorname{Re} f(x) \leqq 1 \text { for all } f \text { in } T\} .
$$

It is known (bipolar theorem) that

$$
\begin{aligned}
S^{00}=\left(S^{0}\right)^{0} & =w^{*}-c l .(\operatorname{co}(S \cup\{0\})) & & \left(S \subset X \subset X^{\prime \prime}\right) \\
\left({ }^{0} T\right)^{0} & =w^{*}-c l .(\operatorname{co}(T \cup\{0\})) & & \left(T \subset X^{\prime}\right) .
\end{aligned}
$$

The following lemma follows easily from Lemma 3.1 and the above comments.

4.1. Lemma. Let $(X, u)$ be a numerical range space. Then

(i) $\operatorname{Dis}(X)^{0}=\mathbb{R}_{0}^{+} D(X)$.

(ii) ${ }^{0}\left(B\left(X^{\prime}\right)+\mathbb{R}_{0}^{+} D(X)\right)=B(X) \cap \operatorname{Dis}(X)$, where $\mathbb{R}_{0}^{+}=\{\lambda \in \mathbb{R}: \lambda \geqq 0\}$.

4.2. Lemma. Let $(X, u)$ be a numerical range space. Then

$$
B\left(X^{\prime \prime}\right) \cap \operatorname{Dis}\left(X^{\prime \prime}\right) \subset w^{*}-c l .(B(X) \cap \operatorname{Dis}(X)) .
$$

Proof. Fix $F$ in $B\left(X^{\prime \prime}\right) \cap \operatorname{Dis}\left(X^{\prime \prime}\right)$. For all $\lambda \geqq 0, f$ in $D(X)$ and $g$ in $B\left(X^{\prime}\right)$ we have

$$
\operatorname{Re} F(g+\lambda f)=\operatorname{Re} F(g)+\lambda \operatorname{Re} F(f) \leqq \operatorname{Re} F(g) \leqq 1,
$$

where we have used the fact that when $X^{\prime}$ is considered as a subspace of $X^{\prime \prime \prime}$ we have $D(X) \subset D\left(X^{\prime \prime}\right)$. Thus

$$
B\left(X^{\prime \prime}\right) \cap \operatorname{Dis}\left(X^{\prime \prime}\right) \subset\left(B\left(X^{\prime}\right)+\mathbb{R}_{0}^{+} D(X)\right)^{0}
$$

By the Banach-Alaoglu theorem and the first part of the lemma above $B\left(X^{\prime}\right)+\mathbb{R}_{0}^{+} D(X)$ is convex and $w^{*}$-closed so that

$$
B\left(X^{\prime}\right)+\mathbb{R}_{0}^{+} D(X)=\left[{ }^{0}\left(B\left(X^{\prime}\right)+\mathbb{R}_{0}^{+} D(X)\right)\right]^{0} .
$$

Now the second part of Lemma 4.1 gives us

$$
B\left(X^{\prime}\right)+\mathbb{R}_{0}^{+} D(X)=(B(X) \cap \operatorname{Dis}(X))^{0}
$$

and we conclude that

$$
B\left(X^{\prime \prime}\right) \cap \operatorname{Dis}\left(X^{\prime \prime}\right) \subset(B(X) \cap \operatorname{Dis}(X))^{00}=w^{*}-c l .(B(X) \cap \operatorname{Dis}(X)) .
$$


4.3. Lemma. Let $(X, u)$ be a numerical range space and $S=\{x \in B(X): M(x)=0\}$. Assume that $\lim _{\alpha \rightarrow 0} \phi_{S}(\alpha)=0$. Then $(X, u)$ is strong. have

Proof. For $x$ in $B(X)$ and $0<\alpha<\frac{1}{2}$ we have clearly $\frac{1}{2} \leqq 1+\alpha M(x)$. If $x=M(x) u$ we

$$
\frac{\|u+\alpha x\|-1}{\alpha}-M(x)=0 .
$$
Otherwise the element $y=\frac{x-M(x) u}{\|x-M(x) u\|}$ belongs to $S$ and writing $\beta=\frac{\alpha\|x-M(x) u\|}{1+\alpha M(x)}$
we have

$$
\begin{aligned}
\frac{\|u+\alpha x\|-1}{\alpha}-M(x) & =(1+\alpha M(x)) \frac{\|u+\beta y\|-1}{\alpha} \\
& =\|x-M(x) u\| \frac{\|u+\beta y\|-1}{\beta} \\
& \leqq 2 \frac{\|u+\beta y\|-1}{\beta} \leqq 2 \phi_{S}(\beta) \leqq 2 \phi_{S}(4 \alpha),
\end{aligned}
$$

where we have used the fact that $\phi_{S}$ is nondecreasing and $\beta \leqq 4 \alpha$.

Since $x$ was arbitrary in $B(X)$ we have proved that

$$
\phi(\alpha) \leqq 2 \phi_{S}(4 \alpha) \text { for } 0<\alpha<\frac{1}{2}
$$

and from $\lim _{\alpha \rightarrow 0} \phi_{S}(\alpha)=0$ we deduce $\lim _{\alpha \rightarrow 0} \phi(\alpha)=0$; that is $(X, u)$ is strong.

4.4. THEOREM. The bidual of a strong numerical range space is a strong numerical range space.

Proof. Let $(X, u)$ be any numerical range space and $\phi$ the function defined in 2.1 . We have clearly

$$
\begin{aligned}
B(X) \cap \operatorname{Dis}(X) & \subset\left\{x \in B(X): \frac{\|u+\alpha x\|-1}{\alpha} \leqq \phi(\alpha)\right\} \\
& \subset\left\{F \in B\left(X^{\prime \prime}\right): \frac{\|u+\alpha F\|-1}{\alpha} \leqq \phi(\alpha)\right\} .
\end{aligned}
$$

The last subset of $X^{\prime \prime}$ (call it $A$ ) is $w^{*}$-closed and so by Lemma 4.2 we have $B\left(X^{\prime \prime}\right) \cap \operatorname{Dis}\left(X^{\prime \prime}\right) \subset A$. Now let $S=\left\{F \in B\left(X^{\prime \prime}\right): M(F)=0\right\}$. We have clearly

$$
S \subset B\left(X^{\prime \prime}\right) \cap \operatorname{Dis}\left(X^{\prime \prime}\right) \subset A
$$

so that

$$
\phi_{S}(\alpha)=\operatorname{Sup}\left\{\frac{\|u+\alpha F\|-1}{\alpha}: F \in S\right\} \leqq \phi(\alpha)
$$


for all $\alpha>0$. Now if $(X, u)$ is strong we have $\lim _{\alpha \rightarrow 0} \phi(\alpha)=0$ so that $\lim _{\alpha \rightarrow 0} \phi_{S}(\alpha)=0$ and $\left(X^{\prime \prime}, u\right)$ is strong in view of Lemma 4.3.

From the theorem above and the main result in Section 3 we deduce the following consequence.

4.5. Corollary. Let $X$ be a Banach space and $u \in S(X)$. If the norm of $X$ is Fréchet differentiable at $u$, then the norm of $X^{\prime \prime}$ is also Fréchet differentiable at $u$.

Proof. Since $(X, u)$ is a strong numerical range space we have by the theorem above that $\left(X^{\prime \prime}, u\right)$ is also strong. We can then apply Theorem 3.4 and Lemma 3.2 to obtain $D\left(X^{\prime \prime}\right)=w^{*}-c l . D(X)$ and so $X^{\prime \prime}$ is smooth at $u$.

5. Relations with upper semicontinuity of the duality mapping. Applications to normed algebras. The duality mapping of a Banach space $X$ is the set-valued mapping $x \rightarrow D(X, x)$ from $S(X)$ into the subsets of $S\left(X^{\prime}\right)$ given by

$$
D(X, x)=\left\{f \in S\left(X^{\prime}\right): f(x)=1\right\} \quad(x \in S(X)) .
$$

Following [8], if $\tau$ is a linear topology on $X^{\prime}$ the duality mapping $D$ of $X$ will be said to be $n-\tau$ upper semicontinuous at a point $x$ in $S(X)$ whenever for all $\tau$-neighbourhoods of zero $V$ in $X^{\prime}$ there is a real positive number $\delta$ such that

$$
D(X, y) \subset D(X, x)+V
$$

holds for all $y$ in $S(X)$ satisfying $\|y-x\|<\delta$. We denote by $n$ (resp. $w$ ) the norm (resp. weak) topology on $X^{\prime}$.

5.1. TheOREM. Let $(X, u)$ be a numerical range space and consider the following statements.

(i) The duality mapping of $X$ is $n-n$ upper semicontinuous at $u$.

(ii) $(X, u)$ is a strong numerical range space.

(iii) The duality mapping of $X$ is $n-w$ upper semicontinuous at $u$. Then (i) $\Rightarrow$ (ii) $\Rightarrow$ (iii).

Proof. (i) $\Rightarrow$ (ii) Fix $\varepsilon>0$ and apply (i) to obtain a $\delta>0$ such that for all $y \in S(X)$ satisfying $\|y-u\|<\delta$ we can find $g \in D(X, y)$ and $f \in D(X, u)$ such that $\|g-f\|<\varepsilon$. Write $\eta=\operatorname{Min}\left\{\frac{\delta}{4}, \frac{1}{2}\right\}$. For $0<\alpha<\eta$ and $x \in B(X)$ we have clearly $\|u+\alpha x\| \geqq \frac{1}{2}$ and

$$
\left\|\frac{u+\alpha x}{\|u+\alpha x\|}-u\right\| \leqq 2\|[1-\|u+\alpha x\|] u+\alpha x\| \leqq 4 \alpha<\delta
$$

so there are $g \in D\left(X, \frac{u+\alpha x}{\|u+\alpha x\|}\right)$ and $f \in D(X, u)$ satisfying $\|g-f\|<\varepsilon$. Then from

$$
\operatorname{Re} g(x)=\frac{1}{\alpha}[\operatorname{Re} g(u+\alpha x)-\operatorname{Re} g(u)] \geqq \frac{\|u+\alpha x\|-1}{\alpha}
$$


and $M(x) \geqq \operatorname{Re} f(x)$ we deduce that

$$
\frac{1}{\alpha}(\|u+\alpha x\|-1)-M(x) \leqq \operatorname{Re} g(x)-\operatorname{Re} f(x) \leqq\|g-f\|<\varepsilon .
$$

We have proved that

$$
\operatorname{Sup}\left\{\frac{\|u+\alpha x\|-1}{\alpha}-M(x): x \in B(X)\right\}<\varepsilon
$$

for $0<\alpha<\eta$; that is $(X, u)$ is strong.

(ii) $\Rightarrow$ (iii) By Theorem $4.4\left(X^{\prime \prime}, u\right)$ is strong. Then by Theorem 3.4 and Lemma 3.2 we have

$$
D\left(X^{\prime \prime}, u\right)=w^{*}-c l . D(X, u) .
$$

This is equivalent to the $n-w$ upper semicontinuity at $u$ of the duality mapping of $X$ [8, Theorem 3.1].

5.2. Remark. The statement (i) in the above theorem is

$$
\forall \varepsilon>0 \exists \delta>0: y \in S(X),\|y-u\|<\delta \Rightarrow D(X, y) \subset D(X, u)+\varepsilon B\left(X^{\prime}\right) .
$$

In fact in the proof of (i) $\Rightarrow$ (ii) we have only used the fact that

$$
\forall \varepsilon>0 \exists \delta>0: y \in S(X),\|y-u\|<\delta \Rightarrow D(X, y) \cap\left[D(X, u)+\varepsilon B\left(X^{\prime}\right)\right] \neq \varnothing .
$$

This condition can be taken as the definition of $n-n$ lower semicontinuity of the duality mapping at $u$ instead of the one used in [8], so obtaining an intermediate condition between (i) and (ii).

5.3. Remark. In view of Theorem 5.1 and [8, Example 1.1] the numerical range space $\left(l^{1}(\mathbb{R}), u\right)$ is strong if and only if the sequence $u$ has only a finite number of nonzero terms (see Example 2.4(ii)).

Next we apply the foregoing results to unital complete normed algebras. The algebraic assumptions are the weakest possible, as the following definition shows.

5.4. Definition. By unital complete normed algebra we mean a Banach space $A$ equipped with a norm-one continuous bilinear mapping $(a, b) \rightarrow a b$ from $A \times A$ into $A$ (the product of $A$ ) and such that there is a norm-one element $I$ in $A$ (the unit of $A$ ) satisfying $I a=a I=a$ for all $a$ in $A$. We remark that the product need not be associative.

5.5. TheоRем. [9, Proposition 4.5]. Let $A$ be a unital complete normed algebra and I the unit of $A$. The duality mapping of $A$ is $n-n$ upper semicontinuous at $I$.

The above theorem together with 5.1 gives the following consequences.

5.6. COROLlary. Let $A$ be a unital complete normed algebra, $\Lambda$ a nonempty set and $\Phi$ a bounded function from $\Lambda$ into $A$. Then

$$
V(\Phi)=\overline{\operatorname{co}} \bigcup_{\lambda \in \Lambda} V(\Phi(\lambda))
$$


Proof. Apply Theorem 2.7 to the strong numerical range space $(A, I)$.

5.7. REMARK. The above result was announced in [11], where it was proved in the power-associative case

5.8. Corollary. Let $A$ be a unital complete normed algebra and I the unit of $A$. Assume that $A$ is the dual space of a Banach space $A_{*}$. Then

$$
V(a)=\left\{a\left(a_{*}\right): a_{*} \in S\left(A_{*}\right), I\left(a_{*}\right)=1\right\}^{-}
$$

for all $a$ in $A$.

Proof. Apply Theorem 3.4 with $X=A_{*}$ and $g=I$.

The class of unital complete normed algebras which are dual Banach spaces is very wide. It includes $W^{*}$-algebras, extremal algebras of $\mathrm{B}$. Bollobás $[2,5], J B W$-algebras [13] and noncommutative $J W^{*}$-algebras [10]. The above corollary appears in [3, Theorem 12.12] for the particular case of $A$ being the bidual of a unital Banach algebra.

For the next corollary we need some notation. The numerical radius $v(x)$ of an element $x$ in a numerical range space $X$ is defined by

$$
v(x)=\operatorname{Max}\{|\lambda|: \lambda \in V(x)\} .
$$

The numerical index $n(X)$ of $X$ is then defined by

$$
n(X)=\operatorname{Inf}\{v(x): x \in S(X)\} .
$$

Equivalently, $n(X)$ is the greatest nonnegative real constant $K$ such that $K\|x\| \leqq$ $v(x)$ for all $x$ in $X$.

5.9. Corollary. Let $(X, u)$ be a numerical range space with $n(X)=1$. Then the duality mapping of $X$ is $n-n$ upper semicontinuous at $u$.

Proof. Each element $x$ in $X$ defines a continuous function $\tilde{x}$ from $D(X)$ into $\mathbb{K}$ by

$$
\bar{x}(f)=f(x) \quad(f \in D(X)) .
$$

In view of the assumption $n(X)=1$ the mapping $x \rightarrow \tilde{x}$ is an isometric linear imbedding of $X$ into the unital Banach algebra $C(D(X), \mathbb{K})$ whose unit is $\tilde{u}$. Now apply Theorem 5.5 and the fact that the property $n-n$ upper semicontinuity of the duality mapping goes down to subspaces.

The assumption $n(X)>0$ on a numerical range space gives important consequences (see [9] for the general case and observe that a number of results about numerical ranges in unital complex Banach algebras follow from the fact that the numerical index of these algebras is greater than or equal to $1 / e[3])$. On the other hand the results in [8] and this paper show that $n-n$ upper semicontinuity of the duality mapping gives also important consequences. In view of the corollary above and the fact that unital complete normed complex algebras satisfy both properties, some relation between these two desirable properties of a numerical range space could be expected. 
Some real algebras, including any real Hilbert space with dimension at least two (see [12, Corollary 28]), and complex Hilbert spaces are examples showing that the numerical index of a numerical range space $(X, u)$ whose duality mapping is $n-n$ upper semicontinuous at $u$ can be zero. On the other hand the following theorem shows that unless $n(X)=1$ the assumption $n(X)>0$ does not imply even the $n-w$ upper semicontinuity of the duality mapping at $u$.

5.10. THEOREM. Let $\rho$ be a real number with $0<\rho<1$. There is a numerical range space $(X, u)$ satisfying

(i) $n(X)=\rho$,

(ii) the duality mapping of $X$ is not $n-w$ upper semicontinuous at $u$.

We need the following lemma which is of independent interest.

5.11. LemMA. Let $\left(X_{\lambda}, u_{\lambda}\right)_{\lambda \in \Lambda}$ be a family of numerical range spaces over the same field $\mathbb{K}$. Consider the Banach space

$$
l^{\infty}\left(X_{\lambda}\right)=\left\{x=\left(x_{\lambda}\right): \operatorname{Sup}\left\{\left\|x_{\lambda}\right\|: \lambda \in \Lambda\right\}<\infty\right\}
$$

with the supremum norm and let $X$ be a closed subspace of $l^{\infty}\left(X_{\lambda}\right)$ containing the element $u=\left(u_{\lambda}\right)$ and the elements $\left(x_{\lambda}\right)$ with $x_{\lambda}=0$ for all but a finite set of $\lambda$ 's. Then the numerical index of $(X, u)$ is

$$
n(X)=\operatorname{Inf}\left\{n\left(X_{\lambda}\right): \lambda \in \Lambda\right\}
$$

Proof. Write $L=\operatorname{Inf}\left\{n\left(X_{\lambda}\right): \lambda \in \Lambda\right\}$ and fix $\lambda_{0}$ in $\Lambda$. The mapping $x \rightarrow x_{\lambda_{0}}$ from $X$ into $X_{\lambda_{0}}$ satisfies $\left\|x_{\lambda_{0}}\right\| \leqq\|x\|$ for all $x$ in $X$ and it maps $u$ into $u_{\lambda_{0}}$ so we have $V\left(x_{\lambda_{0}}\right) \subset V(x)$ for all $x$ in $X$ and

$$
v(x) \geqq v\left(x_{\lambda_{0}}\right) \geqq n\left(X_{\lambda_{0}}\right)\left\|x_{\lambda_{0}}\right\| \geqq L\left\|x_{\lambda_{0}}\right\| .
$$

Since $\lambda_{0}$ was arbitrary we have $v(x) \geqq L\|x\|$ for all $x$ in $X$, so that $n(X) \geqq L$.

Now fix $x_{\lambda_{0}} \in X_{\lambda_{0}}$ and take $x_{\lambda}=0$ for $\lambda \neq \lambda_{0}$. Then $x=\left(x_{\lambda}\right)$ belongs to $X$ and

$$
\begin{aligned}
M(x) & =\lim _{\alpha \rightarrow 0+} \frac{\|u+\alpha x\|-1}{\alpha}=\lim _{\alpha \rightarrow 0+} \operatorname{Max}\left\{0, \frac{1}{\alpha}\left(\left\|u_{\lambda_{0}}+\alpha x_{\lambda_{0}}\right\|-1\right)\right\} \\
& =\operatorname{Max}\left\{0, M\left(x_{\lambda_{0}}\right)\right\} .
\end{aligned}
$$

Replace $x_{\lambda_{0}}$ by $z x_{\lambda_{0}}$ with $z \in \mathbb{K},|z|=1$ in the equality above to obtain

$$
V(x)=\operatorname{co}\left(V\left(x_{\lambda_{0}}\right) \cup\{0\}\right) \text { and } v(x)=v\left(x_{\lambda_{0}}\right) \text {. }
$$

Then $v\left(x_{\lambda_{0}}\right) \geqq n(X)\|x\| \geqq n(X)\left\|x_{\lambda_{0}}\right\|$. Since $x_{\lambda_{0}}$ was an arbitrary element in $X_{\lambda_{0}}$ we have proved that $n(X) \leqq n\left(X_{\lambda_{0}}\right)$, and this is true for all $\lambda_{0}$, so that $n(X) \leqq L$.

Proof of Theorem 5.10. Let $\left(\mu_{k}\right)$ be a sequence with $0<\mu_{k}<1$ for all $k$ and $\left(\mu_{k}\right) \rightarrow 1$. For each natural number $k$ let $X_{k}$ be $\mathbb{R}^{2}$ provided with the absolute norm

$$
|(\xi, \eta)|_{k}=\operatorname{Max}\left\{|\xi|+\rho|\eta|, \mu_{k}|\xi|+|\eta|\right\} \quad(\xi, \eta \in \mathbb{R}) .
$$


Let $X$ be the Banach space of convergent sequences in $\mathbb{R}^{2}$ with the norm

$$
\|x\|=\left\|\left(\left(\xi_{k}, \eta_{k}\right)\right)\right\|=\operatorname{Sup}\left\{\left|\left(\xi_{k}, \eta_{k}\right)\right|_{k}: k \in \mathbb{N}\right\}
$$

and write $u=\left(u_{k}\right)$, where $u_{k}=(1,0)$ for all $k$.

The numerical index of $\left(X_{k}, u_{k}\right)$ is $\rho$ for all $k$ (see [4, Lemma 21.4]). By the lemma above we have $n(X)=\rho$.

A straightforward computation shows that $X^{\prime}$ is the space of sequences $f=$ $\left(\left(a_{k}, b_{k}\right): k=0,1,2, \ldots\right)$ such that the series $\sum_{k=1}^{\infty}\left|\left(a_{k}, b_{k}\right)\right|_{k}^{\prime}$ is convergent, where $|(., .)|_{k}^{\prime}$ denotes the dual norm of $|(., .)|_{k}$ with the norm

$$
\|f\|=\operatorname{Max}\left\{\left|a_{0}\right|,\left|b_{0}\right|\right\}+\sum_{k=1}^{\infty}\left|\left(a_{k}, b_{k}\right)\right|_{k}^{\prime},
$$

the canonical duality being given by

$$
f(x)=a_{0} \xi+b_{0} \eta+\sum_{k=1}^{\infty}\left(a_{k} \xi_{k}+b_{k} \eta_{k}\right)
$$

for $x=\left(\left(\xi_{k}, \eta_{k}\right): k=1,2,3 \ldots\right)$ in $X$ and $(\xi, \eta)=\lim \left(\xi_{k}, \eta_{k}\right)$.

Now by the standard procedure $X^{\prime \prime}$ is the space of bounded sequences $F=$ $\left(\left(c_{k}, d_{k}\right): k=0,1,2, \ldots\right)$ with the norm

$$
\|F\|=\operatorname{Sup}\left\{\left|c_{0}\right|+\left|d_{0}\right|,\left|\left(c_{k}, d_{k}\right)\right|_{k}: k \in \mathbb{N}\right\} .
$$

Let $F_{0}$ in $X^{\prime \prime}$ be given by

$$
F_{0}=((0,0),(0,1),(0,1), \ldots,(0,1), \ldots) .
$$

One easily obtains $V\left(F_{0}\right)=[-1,1]$.

The state space $D(X)$ of $X$ is given by

$$
D(X)=\left\{f=\left(\left(a_{k}, b_{k}\right)\right):\left|b_{0}\right| \leqq a_{0},\left|b_{k}\right| \leqq \rho a_{k}, k \in \mathbb{N}, a_{0}+\sum_{k=1}^{\infty} a_{k}=1\right\}
$$

and so we have

$$
\left\{F_{0}(f): f \in D(X)\right\}^{-}=[-\rho, \rho] \neq[-1,1] .
$$

By Lemma 3.2, $D\left(X^{\prime \prime}\right)$ is not the $w^{*}$-closure of $D(X)$, and so by [8, Theorem 3.1] the duality mapping of $X$ is not $n-w$ upper semicontinuous.

\section{REFERENCES} 1974).

1. S. K. Berberian, Lectures in functional analysis and operator theory, (Springer-Verlag,

2. B. Bollobás, The numerical range in Banach algebras and complex functions of exponential type, Bull. London Math. Soc. 3 (1971), 27-33. 
3. F. F. Bonsall, and J. Duncan, Numerical ranges of operators on normed spaces and of elements of normed algebras, (London Math. Soc. Lecture Note Series 2, Cambridge, 1971).

4. F. F. Bonsall, and J. Duncan, Numerical ranges II, (London Math. Soc. Lecture Note Series 10, Cambridge, 1973).

5. M. J. Crabb, J. Duncan, and C. M. McGregor, Some extremal problems in the theory of numerical ranges, Acta Math. 128 (1972), 123-142.

6. J. Diestel, Geometry of Banach spaces. Selected topics. Lecture Notes in Mathematics No. 48, (Springer-Verlag, 1975).

7. N. Dunford, and J. T. Schwartz, Linear operators. Part I, (Interscience Publishers, New York, 1958).

8. J. R. Giles, D. A. Gregory, and Brailey Sims. Geometrical implications of upper semi-continuity of the duality mapping on a Banach Space, Pacific J. Math. 79 (1978), 99-108.

9. J. Martinez, J. F. Mena, R. Payá, and A. Rodríguez, An approach to numerical ranges without Banach algebra theory. Illinois J. Math. (to appear).

10. R. Payá, J. Perez, and A. Rodríguez, Noncommutative Jordan $C^{*}$-algebras. Manuscripta Math. 37 (1982), 87-120.

11. A. Rodríguez, A Vidav-Palmer theorem for Jordan $C^{*}$-algebras and related topics. $J$. London Math. Soc. (2) 22 (1980), 318-332.

12. A. Rodríguez, Non-associative normed algebras spanned by hermitian elements. Proc. London Math. Soc. (3) 47 (1983), 258-274.

13. F. W. Shultz, On normed Jordan algebras which are Banach dual spaces. J. Functional Analysis 31 (1979), 360-376.

Departamento de TeORia de Funciones

Facultad de Ciencias

UNIVERSIDAD DE GRANADA

SPAIN 